Pacific Journal of Mathematic 


\title{
A CHARACTERIZATION OF PERFECT RINGS
}

\author{
Vlastimil Dlab
}

J. P. Jans has shown that if a ring $R$ is right perfect, then a certain torsion in the category $\operatorname{Mod} R$ of left $R$-modules is closed under taking direct products. Extending his method, J. S. Alin and E. P. Armendariz showed later that this is true for every (hereditary) torsion in $\operatorname{Mod} R$. Here, we offer a very simple proof of this result. However, the main purpose of this paper is to present a characterization of perfect rings along these lines: A ring $R$ is right perfect if and only if every (hereditary) torsion in $\operatorname{Mod} R$ is fundamental (i.e., derived from "prime" torsions) and closed under taking direct products; in fact, then there is a finite number of torsions, namely $2^{n}$ for a natural number $n$. Finally, examples of rings illustrating that the above characterization cannot be strengthened are provided. Thus, an example of a ring $R_{1}$ is given which is not perfect, although there are only fundamental torsions in $\operatorname{Mod} R_{1}$, and only $4=2^{2}$ of these. Furthermore, an example of a ring $R_{2 *}$ is given which is not perfect and which, at the same time, has the property that there is only a finite number (namely, 3) of (hereditary) torsions in $\operatorname{Mod} R_{2 *}$ all of which are closed under taking direct products. Moreover, the ideals of $R_{2 *}$ form a chain (under inclusion) and $\operatorname{Rad} R_{2 *}$ is a nil idempotent ideal; all the other proper ideals are nilpotent and $R_{2 *}$ can be chosen to have a (unique) minimal ideal.

In what follows, $R$ stands always for a ring with unity, $\mathscr{L}$ for the set of all left ideals of $R$ and $\operatorname{Mod} R$ for the category of all (unital) left $R$-modules and $R$-homomorphisms. Given $L \in \mathscr{L}$ and $\rho \in R, L: \rho$ denotes the (right) ideal-quotient of $L$ by $\rho$, i.e., the left ideal of all $\chi \in R$ such that $\chi \rho \in L$. We shall call a subset $\mathscr{K}$ of $\mathscr{L}$ a $Q$-set if it is closed with respect to this operation, i.e., if $K \in \mathscr{C}$ and $\rho \in R$ implies $K: \rho \in \mathscr{L}$; obviously, $\mathscr{L}$ and $\{R\}$ are the greatest and the least $Q$-sets, respectively. Thus, a topologizing idempotent filter (briefly, a $T$-set) of left ideals of P. Gabriel [4] is a $Q$-set $\mathscr{C}$ satisfying, in addition to the filter properties, also the following "radical" condition: If $L$ is a left ideal of $R$ such that $L: \kappa \in \mathscr{K}$ for every element $\kappa$ of $K \in \mathscr{K}$, then $L \in \mathscr{K}$, as well.

By a torsion $T$ in $\operatorname{Mod} R$ we shall always understand a hereditary torsion; thus, a torsion $T$ in $\operatorname{Mod} R$ is a full subcategory of $\operatorname{Mod} R$ such that

(a) $T$ is closed under taking submodules,

(b) for every $M \in \operatorname{Mod} R$, there is the greatest submodule (the $T$ torsion part) $T(M)$ of $M$ belonging to $T$ and 
(c) $T(M / T(M))=0$ for every $M \in \operatorname{Mod} R$.

As a consequence, every torsion in $\operatorname{Mod} R$ is closed under taking quotients, direct sums and inductive limits. There is a one-to-one correspondence between the torsions in $\operatorname{Mod} R$ and the $T$-sets of left ideals of $R$ :

If $\mathscr{K}$ is a $T$-set, then the class $T(\mathscr{K})$ of all $R$-modules whose elements have orders from $\mathscr{K}$ is a torsion in $\operatorname{Mod} R$; on the other hand, if $T$ is a torsion in $\operatorname{Mod} R$, then the $T$-set $\mathscr{K}(T)=\{L \mid L \in \mathscr{L}$ and $R \bmod L \in T\}$ satisfies $T=T[\mathscr{K}(T)]$. Given an $R$-module $M$, let us always denote the $T$-torsion part of it by $T(M)$.

Thus, given a torsion $T$, we can define the following two-sided ideals $I_{T}$ and $J_{T} \supseteqq I_{T}$ of $R$ :

$$
I_{T}=\bigcap_{L \in \mathscr{R}(T)} L
$$

and

$$
J_{T} / I_{T}=T\left(R / I_{T}\right) .
$$

Using this notation, we can prove easily

Proposition 1. The following four statements are equivalent:

(i ) A torsion $T$ in $\operatorname{Mod} R$ is closed under taking direct products.

(ii) For every subset $\mathscr{S}$ of $\mathscr{K}(T)$,

$$
\bigcap_{L \in \mathcal{L}} L \in \mathscr{K}(T) \text {. }
$$

$$
\begin{gathered}
I_{T} \in \mathscr{\mathscr { K }}(T) \\
J_{R}=R .
\end{gathered}
$$

Proof. The equivalence of (ii), (iii), and (iv) is trivial. Also the implication (ii) $\rightarrow$ (i) follows easily; for, the order of an element of a direct product is evidently the intersection of the orders of its components. Finally, in order to show that (i) $\rightarrow$ (iv), we consider the monogenic submodule of the direct product

$$
\prod_{L \in \mathscr{K}^{\prime}(T)} R \bmod L
$$

generated by the element whose components are generators of $R \bmod L$; it is obviously $R$-isomorphic to $R / I_{T}$.

Proposition 2. Let every proper $(i . e ., \quad \neq R$ ) two-sided ideal $J$ of $R$ satisfy the following condition: There is $\kappa \notin J$ such that, for every $\rho \in R$ with $\rho \kappa \notin J$, there exists $\sigma \in R$ with $\sigma \rho \kappa=\kappa$. Then every torsion in $\operatorname{Mod} R$ is closed under taking direct products. 
Proof. Let $T$ be a torsion and $J_{T}$ the two-sided ideal defined above. Assume that $J_{T} \neq R$. Thus, there exists $\kappa \notin J_{T}$ with the properties stated in our assumption. Since

$$
\bigcap_{L \in \mathscr{T}(T)} L=I_{T} \subseteq J_{T},
$$

there is $L_{0} \in \mathscr{K}(T)$ such that $\kappa \notin L_{0}$. Hence

$$
L_{0}: \kappa=\left(R \kappa \cap L_{0}\right): \kappa \subseteq J_{T}: \kappa,
$$

and therefore $J_{T}: \kappa \in \mathscr{K}(T)$, in contradiction to the fact that $R / J_{T}$ has no nonzero element of order belonging to $\mathscr{K}(T)$. Consequently, $J_{T}=R$ and Proposition 2 follows in view of Proposition 1.

THEOREM A. If a ring $R$ satisfies the minimum condition on principal left ideals, i.e., if $R$ is right perfect (cf. H. Bass [2]), then every torsion in $\operatorname{Mod} R$ is closed undertaking direct products.

Proof. Given an ideal $J \neq R$, consider the (nonempty) set of all principal left ideals which are not contained in $J$; take a minimal element $K$ of this set, $\kappa \in K \backslash J$ and apply Proposition 2.

REMARK 1. We can see easily that if $R$ satisfies the minimum condition on principal left ideals, then every $R$-module $M$ has a nonzero socle; the latter property is, in turn, obviously equivalent to either of the following two statements:

(i) Every monogenic $R$-module has a nonzero socle.

(ii) For every proper left ideal $L$ of $R$, there is $\rho \in R \backslash L$ such that $L: \rho \neq R$ is maximal in $R$.

Before we proceed to establish the characterization of perfect rings, left us introduced the following convenient notation and terminology. Denote by $\mathscr{W} \cong \mathscr{L}$ the $Q$-set of all maximal left ideals of $R$ ( $R$ itself including). Obviously, for every $W \in \mathscr{W}, W \neq R$, the subset

$$
\{W: \rho \mid \rho \in R\}
$$

is a minimal $Q$-set contained in $\mathscr{W}$. Denoting by $\mathscr{W}_{\omega}, \omega \in \Omega$, all such (distinct) minimal $Q$-sets, it is easy to see that $\left\{\mathscr{W}_{\omega} \mid \omega \in \Omega\right\}$ is a covering of $\mathscr{W}$, i.e.,

$$
\mathscr{Y}=\bigcup_{\omega \in \Omega} \mathscr{W}_{\omega} \text { and } \mathscr{W}_{\omega_{1}} \cap \mathscr{W}_{\omega_{2}}=\{R\} \text { for } \omega_{1} \neq \omega_{2} .
$$

Furthermore, for every $\Omega_{1} \subseteq \Omega$, put

$$
\mathscr{W}_{\Omega_{1}}=\bigcap_{\omega \in \Omega_{1}} \mathscr{W}_{\omega} ;
$$


of course, $\mathscr{W}=\mathscr{W}_{\Omega}$ and $\mathscr{W}_{\omega}=\mathscr{W}_{\{\Omega\}}$ for each $\omega \in \Omega$. Now, for every $\Omega_{1} \leqq \Omega$, denote the smallest $T$-set containing $\mathscr{W}_{\Omega_{1}}$ by $\mathscr{W}_{\Omega_{1}}^{*}$. It can be easily shown (cf. [3]) that $\mathscr{W}_{\Omega_{1}}^{*}$ is the unique $T$-set $\sim$-equivalent to $\mathscr{W}_{\Omega_{1}}$ in the sense that, for every proper left ideal $L \in \mathscr{W}_{\Omega_{1}}^{*}$,

$$
\{L: \rho \mid \rho \in R\} \cap \mathscr{W}_{\Omega_{1}} \neq\{R\} .
$$

As a consequence,

$$
\mathscr{W}_{\Omega_{1}}^{*} \cap \mathscr{W}=\mathscr{W}_{\Omega_{1}} .
$$

Let us call the torsions $T\left(\mathscr{W}_{\omega}^{*}\right), \omega \in \Omega$, the prime torsions in $\operatorname{Mod} R$ and, more generally, torsions $T\left(\mathscr{W}_{\Omega_{1}}^{*}\right)$ corresponding to the subsets $\Omega_{1}$ of $\Omega$, the fundamental torsions (i.e., derived from prime ones) in $\operatorname{Mod} R$.

On the basis of the above characterization of the $T$-sets $\mathscr{W}_{a_{1}}^{*}$, one can derive very easily the following well-known

Proposition 3. For any ring $R$, all the fundamental torsions $T\left(\mathscr{W}_{\Omega_{1}}^{*}\right)$ in $\operatorname{Mod} R$ are distinct and form a lattice ideal of the complete lattice of all torsions in $\operatorname{Mod} R$, which is isomorphic to the lattice $2^{\Omega}$ of all subsets of $\Omega$.

Proof. In order to complete the proof we need only to show that every torsion $T$ in $\operatorname{Mod} R$ contained in $T\left(\mathscr{V}^{*}\right)$ is fundamental. But this follows from the fact that the $T$-set $\mathscr{C}(T) \subseteq \mathscr{W}^{*}$ is evidently $\sim$-equivalent to $\mathscr{K}(T) \cap \mathscr{W}$ and since $\mathscr{K}(T) \cap \mathscr{W}=\mathscr{W} \Omega_{\Omega_{0}}$ for a suitable $\Omega_{0} \subseteq \Omega$, we have, in view of the fact that there is unique $T$ set $\sim$-equivalent to $\mathscr{V}_{\Omega_{0}}$,

$$
\mathscr{K}(T)=\mathscr{W}_{\Omega_{0}}^{*},
$$

as required.

REMARK 2. We can see easily that the assertion that every torsion in $\operatorname{Mod} R$ is fundamental is equivalent to the assertion that $\mathscr{Y}^{*}=\mathscr{L}$, which in turn is equivalent to any of the statements of the previous Remark 1 (for, $\mathscr{Y}^{*} \sim \mathscr{W}$ ).

Now, let us formulate the following

THeOREM B. Let $R$ be a ring such that every fundamental torsion in $\operatorname{Mod} R$ is closed under taking direct products. Then $R / \operatorname{Rad} R$ is semisimple (i.e., artinian); in particular, $\Omega$ is finite.

Proof. For each $\omega \in \Omega$, put

$$
W_{\omega}^{0}=\bigcap_{W \in \mathscr{V}} W
$$


and notice that the intersection

$$
\operatorname{Rad} R=\bigcap_{\omega \in \Omega} W_{\omega}^{0}
$$

is, according to Proposition 3, irredundant. For, $\mathscr{W}_{\omega}^{*}$ (for each $\omega \in \Omega$ ) and $\mathscr{W}^{*}$ are the smallest $T$-sets containing $W_{\omega}^{0}$ and $\operatorname{Rad} R$, respectively. In order to prove our theorem, it is sufficient to show that the socle of $R / \operatorname{Rad} R$ is the whole quotient $\operatorname{ring} R / \operatorname{Rad} R$; for, $R / \operatorname{Rad} R$ is a ring with unity. First, observe that, in view of the fact that $\operatorname{Rad} R \in \mathscr{W}^{*}$, the socle of $R / \operatorname{Rad} R$ is essential in $R / \operatorname{Rad} R$ in the sense that it intersect every nonzero left ideal of $R / \operatorname{Rad} R$ nontrivially. Write

$$
S / \operatorname{Rad} R=\text { Socle }(R / \operatorname{Rad} R)
$$

with the two-sided ideal $S \supseteqq \operatorname{Rad} R$ of $R$ and assume

$$
S \neq R \text {. }
$$

Then, there is a (proper) maximal left ideal $W$ of $R$ such that

$$
S \subseteq W \subset R ;
$$

and, $W \in \mathscr{W}_{\omega_{1}}$ for a suitable $\omega_{1} \in \Omega$. Moreover, clearly

$$
S \subseteq W_{\omega_{1}}^{0} .
$$

Hence, since $\bigcap_{\omega \in \Omega} W_{\omega}^{0}$ is irredundant,

$$
\bigcap_{\substack{\omega \in \Omega \\ \omega \neq \omega_{1}}} W_{\omega}^{0} \neq\left(\bigcap_{\substack{\omega \in \Omega \\ \omega \neq \omega_{1}}} W_{\omega_{1}}^{0}\right) \cap W_{\omega_{1}}^{0}=\operatorname{Rad} R
$$

on the other hand, since $\operatorname{Rad} R \subseteq S \subseteq W_{\omega_{1}}^{0}$,

$$
\left(\bigcap_{\substack{\omega \in Q \\ \omega \neq \omega_{1}}} W_{\omega}^{0}\right) \cap S=\operatorname{Rad} R
$$

and thus

$$
\bigcap_{\substack{\omega \in \Omega \\ \omega \neq \omega_{1}}} W_{\omega}^{0}=\operatorname{Rad} R
$$

a contradiction.

The proof of the theorem is completed.

Now, the main result of the present paper, namely the characterization of perfect rings, follows straight forward from Theorem A, Remarks 1 and 2, Theorem $\mathrm{B}$ and the fact that a (right) perfect rings can be characterized as a ring $R$ with unity such that every (left) $R$ module has a nonzero socle and that $R / \operatorname{Rad} R$ is $\operatorname{artinian~(H.~Bass~[2]):~}$ 
COROLLARY. A ring $R$ is right perfect if and only if all torsions in $\operatorname{Mod} R$ are fundamental and are closed under taking direct products.

In conclusion, let us remark that the above characterization cannot be strengthened, even if we take into account the additional condition that there is a finite number of fundamental torsions in $\operatorname{Mod} R$ (the fact which is a consequence of our characterization). To show this, we present the following two examples of rings (which can easily be generalized):

ExAmple 1. Let $N$ be the set of all natural numbers, $F$ a field. Denote by $R_{1}=R_{1}\left(\boldsymbol{\aleph}_{0}, F\right)$ the ring of all countable "bounded" matrices over $F$, i.e., the ring of all functions $f: N \times N \rightarrow F$ satisfying the condition that there is a natural number $n_{f}$ such that

$$
f(i, j)=0 \quad \text { for } i \neq j, i>n_{f} \text { or } j>n_{f}
$$

and

$$
f(i, i)=f\left(n_{f}+1, n_{f}+1\right) \quad \text { for all } i>n_{f},
$$

with matrix addition and multiplication. It is easy to verify that, for every $n \in N$,

$$
C_{n}=\left\{f \mid f \in R_{1} \text { and } f(i, j)=0 \text { for } j \neq n\right\}
$$

are minimal left ideals in $R_{1}$ and that the socle

$$
S=\bigoplus_{n \in N} C_{n}
$$

of $R_{1}$ is a (two-sided) maximal ideal in $R_{1}$; obviously, $R_{1} / S \cong F$. Furthermore, $\mathscr{W}_{1}^{\prime}=\left\{S, R_{1}\right\}$ is a minimal $Q$-set of left ideals of $R_{1}$. Also, for every $n \in N$, the left ideals

$$
W_{n}=\left\{f \mid f \in R_{1} \text { and } f(i, n)=0\right\}
$$

are maximal in $R_{1}$ and belong to the same minimal $Q$-set $\mathscr{W}_{2}^{\prime}$. It is easy to see that the set of all maximal left ideals of $R_{1}$

$$
\mathscr{W}=\mathscr{W}_{1} \cup \mathscr{W}_{2}
$$

and that there are 4 torsions in $\operatorname{Mod} R$, all of them fundamental, namely

$$
0=T(\{R\}), T\left(\mathscr{H}_{1}^{*}\right), T\left(\mathscr{W}_{2}^{*}\right) \text { and } \operatorname{Mod} R=T\left(\mathscr{W}^{*}\right) .
$$

Only $T\left(\mathscr{W}_{2}^{*}\right)$ is not closed under taking direct products. Of course, $R_{1}$ is not perfect.

EXAMPLE 2. Denote by $Q^{+}$the set of all nonnegative rational 
numbers endowed with the usual order $\leqq$. Let $F$ be a field. Denote by $R_{2}=R\left(Q^{+}, F\right)$ the ring of all functions $f: Q^{+} \rightarrow F$ such that the support

$$
\operatorname{Sup} f=\left\{r \mid r \in Q^{+} \text {and } f(r) \neq 0\right\}
$$

is contained in a well-ordered (with respect to $\leqq$ ) subset of $Q^{+}$which has no finite limit point, with the addition and multiplication defined by

$$
\left(f_{1}+f_{2}\right)(r)=f_{1}(r)+f_{2}(r)
$$

and

$$
\left(f_{1} * f_{2}\right)(r)=\sum_{\substack{t \in Q^{+} \\ t \leqq r}} f_{1}(t) \cdot f_{2}(r-t)
$$

respectively.

It is a matter of routine to verify that $R_{2}$ is a (commutative) ring. Now, for every $f \in R_{2}$, denote by $r_{f}$ the least nonzero rational number such that $f\left(r_{f}\right) \neq 0$. Moreover, for every $t \in Q^{+}$, denote by $f^{(t)}$ the function of $R_{2}$ defined by

$$
f^{(t)}(r)= \begin{cases}1 & \text { for } r=t \\ 0 & \text { otherwise }\end{cases}
$$

Now, we can see easily that, for every $f \in R_{2}$,

$$
f=f^{\left(r_{f}\right)} * \bar{f},
$$

where $\bar{f}(r)=f\left(r+r_{f}\right)$ for $r \in Q^{+}$(and thus, $r_{\bar{f}}=0$ ). First, we are going to prove the following

LEMmA. If $\bar{f} \in R_{2}$ such that $r_{\bar{f}}=0$, then there is $\bar{g} \in R_{2}$ satisfying

$$
\bar{f} * \bar{g}=f^{(0)}\left(=\text { unity of } R_{2}\right) \text {. }
$$

Proof. In order to ease the technical difficulties of the proof, observe first that having a well-ordered subset $S$ of $Q^{+}$with no finite limit point, we can consider the subsemigroup $\bar{S}$ of $Q^{+}$generated by $S: \bar{S}$ is again well-ordered and has no finite limit point. Hence, we may consider, for a moment, that our function $\bar{f}$ is defined on a wellordered subsemigroup $\bar{S}$ of $Q^{+}$with no limit point and try to find $\bar{g}$ defined on the same set $\bar{S}$, i.e., with $\operatorname{Sup} \bar{g} \subseteq \bar{S}$. Write

$$
S=\left\{r_{i}\right\}_{i=0}^{\infty} \text { with } 0=r_{0}<r_{1}<r_{2}<\cdots<r_{n}<\cdots \text {. }
$$

Let us proceed by induction: Denoting by $\bar{g}_{1}$ the function defined by 


$$
\bar{g}_{1}(0)=[\bar{f}(0)]^{-1}, \bar{g}_{1}\left(r_{1}\right)=-[\bar{f}(0)]^{-2} \cdot f\left(r_{1}\right) \text { and } \bar{g}_{1}(r)=0 \text { otherwise, }
$$

we can see easily that

where

$$
\bar{f} * \bar{g}_{1}=f^{(0)}+h_{1},
$$

$$
\operatorname{Sup} \bar{g}_{1} \subseteq\left\{r_{i}\right\}_{i=0}^{1} \text { and } \operatorname{Sup} h_{1} \subseteq\left\{r_{i}\right\}_{i=2}^{\infty} \text {. }
$$

Assuming that, for a natural $n \geqq 1$, we have $\bar{g}_{n} \in R_{2}$ and $h_{n} \in R_{2}$ with

$$
\operatorname{Sup} \bar{g}_{n} \subseteq\left\{r_{i}\right\}_{i=0}^{n} \text { and } \operatorname{Sup} h_{n} \subseteq\left\{r_{i}\right\}_{i=n+1}^{\infty}
$$

such that

$$
\bar{f} * \bar{g}_{n}=f^{(0)}+h_{n},
$$

let us define

$$
\bar{g}_{n+1}=\bar{g}_{n}+g_{n+1},
$$

where

$$
g_{n+1}\left(r_{n+1}\right)=-[\bar{f}(0)]^{-1} h_{n}\left(r_{n+1}\right) \text { and } g_{n+1}(r)=0 \text { otherwise. }
$$

Then,

$$
\bar{f} * \bar{g}_{n+1}=\bar{f} * \bar{g}_{n}+\bar{f} * g_{n+1}=f^{(0)}+h_{n}+\bar{f} * g_{n+1}
$$

and, writing

$$
h_{n+1}=h_{n}+\bar{f} * g_{n+1},
$$

we can easily check that

$$
\operatorname{Sup} h_{n+1} \subseteq\left\{r_{i}\right\}_{i=n+2}^{\infty} .
$$

For,

$$
h_{n+1}(r)=\left(\bar{f} * g_{n+1}\right)(r)=\sum_{0 \leqq t \leqq r} \bar{f}(t) g_{n+1}(r-t)=0 \quad \text { for } r<r_{n+1}
$$

and

$$
\begin{aligned}
h_{n+1}\left(r_{n+1}\right) & =h_{n}\left(r_{n+1}\right)+\sum_{0 \leqq t \leqq r_{n+1}} \bar{f}(t) g_{n+1}\left(r_{n+1}-t\right) \\
& =h_{n}\left(r_{n+1}\right)+\bar{f}(0) g_{n+1}\left(r_{n+1}\right)=h_{n}\left(r_{n+1}\right)-h_{n}\left(r_{n+1}\right)=0,
\end{aligned}
$$

as required.

Finally, to complete the proof of our lemma, denote by $\bar{g}$ the function defined by

$$
\bar{g}(r)= \begin{cases}g_{i}\left(r_{i}\right) & \text { for } r=r_{i}, i=0,1, \cdots \\ 0 & \text { elsewhere }\end{cases}
$$


Then,

$$
\bar{f} * \bar{g}=f^{(0)}
$$

for, if $i=1,2, \cdots$

$$
\begin{aligned}
(\bar{f} * \bar{g})\left(r_{i}\right) & =\left(\bar{f} *\left[\bar{g}_{i}+\left(\bar{g}-\bar{g}_{i}\right)\right]\right)\left(r_{i}\right) \\
& =\left(\bar{f} * \bar{g}_{i}\right)\left(r_{i}\right)+\left[\bar{f} *\left(\bar{g}-\bar{g}_{i}\right)\right]\left(r_{i}\right) \\
& =\left(f^{(0)}+h_{i}\right)\left(r_{i}\right)+\left[\bar{f} *\left(\bar{g}-\bar{g}_{i}\right)\right]\left(r_{i}\right) \\
& =0+\sum_{0 \leq t \leq r_{i}} \bar{f}(t)\left(\bar{g}-\bar{g}_{i}\right)\left(r_{i}-t\right) \\
& =0 .
\end{aligned}
$$

As a consequence, $f \in R_{2}$ is a unit in $R_{2}$ if and only if $r_{f}=0$. Moreover, for every $r \in Q^{+}$, there exist two ideals

$$
\bar{I}_{r}=\left\{f \mid f \in R_{2} \text { and } r_{f} \geqq r\right\}
$$

and

$$
I_{r}=\left\{f \mid f \in R_{2} \text { and } r_{f}>; r\right\} ;
$$

these are all ideals of $R_{2}$. Notice that,

$$
I_{r} \subset \bar{I}_{r}
$$

and that

$$
r_{1}<r_{2} \text { implies } I_{r_{1}}^{\prime} \supset \bar{I}_{r_{2}}^{\prime}
$$

in particular,

$$
\bar{I}_{0}=R_{2} \text { and } I_{0}=\operatorname{Rad} R_{2} .
$$

It is also easy to see that there are no divisors of zero in $R_{2}$ and that

$$
\left(\operatorname{Rad} R_{2}\right)^{2}=\operatorname{Rad} R_{2} \text {. }
$$

For, if $f \in \operatorname{Rad} R_{2}$, then $r_{f}>0$ and obviously,

$$
f=f^{\left((1 / 2) r_{f}\right)} * g,
$$

where

$$
g(r)=f\left(r+\frac{1}{2} r_{f}\right) \quad \text { for } r \in Q^{+} ;
$$

here, both $f^{\left((1 / 2) r_{g}\right)}$ and $g$ evidently belong to $\operatorname{Rad} R_{2}$.

Finally, given a positive rational number $q$, define

$$
R_{2 q}=R_{2} / I_{q}
$$

(similarly, we can consider $\bar{R}_{2 q}=R_{2} / \bar{I}_{q}$ ). It is easy to see that 


$$
\operatorname{Rad} R_{2 q} \cong I_{0} / I_{q}
$$

satisfies again

$$
\left(\operatorname{Rad} R_{2 q}\right)^{2}=\operatorname{Rad} R_{2 q},
$$

but that every other proper ideal (which is isomorphic to either $I_{r} / I_{q}$ or $I_{r} / I_{q}$ for $\left.r \geqq q\right)$ is nilpotent; besides,

$$
\text { Socle }\left(R_{2 q}\right) \cong \bar{I}_{q} / I_{q} \text {. }
$$

Thus, there are only three torsions in Mod $R_{2 q}$, namely

$$
0=T(\{R\}), T\left(\left\{R_{2 q}, \operatorname{Rad} R_{2 q}\right\}\right) \text { and } \operatorname{Mod} R_{2 q}=T\left(\mathscr{L}^{R_{2 q}}\right) .
$$

All of them are evidently closed under taking direct products; but, only the first two are fundamental. And, $R_{2 q}$ is not perfect.

\section{REFERENCES}

1. J. S. Alin and E. P. Armendariz, TTF-classes and $E(R)$-torsion modules (to appear).

2. H. Bass, Finistic dimension and homological generalization of semi primary rings, Trans. Amer. Math. Soc. 95 (1960), 466-488.

3. V. Dlab, Distinguished sets of ideals of a ring, Czechoslovak Math. J. 18/93 (1968), 560-567.

4. P. Gabriel, Des catégories abéliennes, Bull. Soc. Math. France 90 (1962), 323-448.

5. J. P. Jans, Some aspects of torsion, Pacific J. Math. 15 (1965), 1249-1259.

Received April 4, 1969.

Carleton University

Ottawa, Ontario, Canada 


\section{PACIFIC JOURNAL OF MATHEMATICS}

\section{EDITORS}

H. SAMELSON

Stanford University

Stanford, California 94305

\section{RichaRd PIERCe}

University of Washington

Seattle, Washington 98105
J. DUGUNDJI

Department of Mathematics

University of Southern California

Los Angeles, California 90007

RICHARD ARENS

University of California

Los Angeles, California 90024

\section{ASSOCIATE EDITORS}

E. F. BECKENBACH

B. H. NeUManN

F. WOLF

K. YosHIDA

\section{SUPPORTING INSTITUTIONS}

UNIVERSITY OF BRITISH COLUMBIA CALIFORNIA INSTITUTE OF TECHNOLOGY UNIVERSITY OF CALIFORNIA MONTANA STATE UNIVERSITY

UNIVERSITY OF NEVADA

NEW MEXICO STATE UNIVERSITY

OREGON STATE UNIVERSITY

UNIVERSITY OF OREGON

OSAKA UNIVERSITY

UNIVERSITY OF SOUTHERN CALIFORNIA
STANFORD UNIVERSITY

UNIVERSITY OF TOKYO

UNIVERSITY OF UTAH

WASHINGTON STATE UNIVERSITY

UNIVERSITY OF WASHINGTON

${ }^{*} \quad{ }^{*} \quad{ }^{*}$
AMERICAN MATHEMATICAL SOCIETY
CHEVRON RESEARCH CORPORATION
TRW SYSTEMS
NAVAL WEAPONS CENTER




\section{Pacific Journal of Mathematics}

\section{Vol. 33, No. $1 \quad$ March, 1970}

Mir Maswood Ali, On some extremal simplexes ................... 1

Silvio Aurora, On normed rings with monotone multiplication........... 15

Silvio Aurora, Normed fields which extend normed rings of integers....... 21

John Kelly Beem, Indefinite Minkowski spaces..................... 29

T. F. Bridgland, Trajectory integrals of set valued functions ........... 43

Robert Jay Buck, A generalized Hausdorff dimension for functions and sets ......................................... 69

Vlastimil B. Dlab, A characterization of perfect rings . . . . . . . . . . . . 79

Edward Richard Fadell, Some examples in fixed point theory ............ 89

Michael Benton Freeman, Tangential Cauchy-Riemann equations and uniform approximation ............................. 101

Barry J. Gardner, Torsion classes and pure subgroups ................ 109

Vinod B. Goyal, Bounds for the solution of a certain class of nonlinear

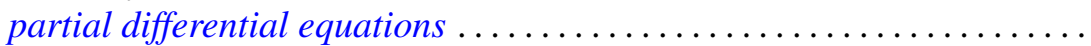

Fu Cheng Hsiang, On C, 1 summability factors of Fourier series at a given

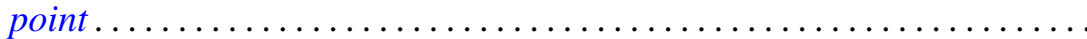

Lawrence Stanislaus Husch, Jr., Homotopy groups of PL-embedding

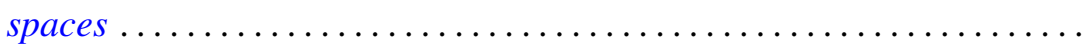

Daniel Ralph Lewis, Integration with respect to vector measures..........

Marion-Josephine Lim, $\mathscr{L}-2$ subspaces of Grassmann product spaces

Stephen J. Pierce, Orthogonal groups of positive definite multilinear functionals

W. J. Pugh and S. M. Shah, On the growth of entire functions of bounded index.

Siddani Bhaskara Rao and Ayyagari Ramachandra Rao, Existence of triconnected graphs with prescribed degrees . . .

Ralph Tyrrell Rockafellar, On the maximal monotonicity of subdifferential

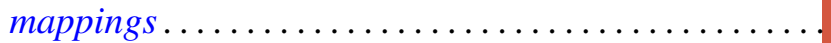

R. Shantaram, Convergence of a sequence of transformations of distribution functions. II ...............................

Julianne Souchek, Rings of analytic functions..............

Ted Joe Suffridge, The principle of subordination applied to functions of several variables...

Wei-lung Ting, On secondary characteristic classes in cobordism

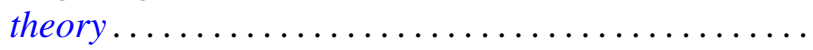

Pak-Ken Wong, Continuous complementors on $B^{*}$-algebras ...

Miyuki Yamada, On a regular semigroup in which the idempotents form a band. 